\title{
The NPS Phenomenon and the Deep Web: Trends Analyses and Internet Snapshots
}

\author{
Ahmed Al-Imam ${ }^{1,2} \&$ Ban A. AbdulMajeed ${ }^{3}$ \\ ${ }^{1}$ Department of Postgraduate Medicine, School of Life and Medical Sciences, University of Hertfordshire, United \\ Kingdom \\ ${ }^{2}$ Department of Anatomy and Cellular Biology, Faculty of Medicine, University of Baghdad, Iraq \\ ${ }^{3}$ Department of Pathology and Forensic Medicine, College of Medicine, Al-Nahrain University, Iraq \\ Correspondence: Dr Ahmed Al-Imam, House 18/5, Al-Akhtal Street, District 318, Al-Adhamyia, 10053, Baghdad, \\ Iraq. E-mail: tesla1452@gmail.com; a.m.al-imam@herts.ac.uk
}

Received: July 25, 2017 Accepted: August 17, 2017 Online Published: September 18, 2017

doi:10.5539/gjhs.v9n11p71 URL: https://doi.org/10.5539/gjhs.v9n11p71

\begin{abstract}
Background: In relation to the phenomenon of novel psychoactive substances, activities on the surface web represent only the tip of the iceberg. The majority of the electronic commerce (e-commerce) activities exist on the deep web and the darknet. Observational analytic studies are failing to keep pace with these activities; these studies are either obsolete beyond the point in time of the taken internet snapshot or highly-consuming for resources including time, funding, and manpower.
\end{abstract}

Materials and Methods: Cross-sectional and retrospective analyses via multiple Internet snapshots were carried out across Google Trends database and the e-markets on the darknet. Google Trends were scanned retrospectively (2012-2016) for keywords specific to the deep web in an aim to estimate and geo-map of the attentiveness (interest) of surface web users in the deep web and its illicit activities.

Results: The attentiveness of surface web users in the deep web was noticed to be incremented during 2013 and 2014; the top ten contributing countries were Norway, Germany, Denmark, Austria, Poland, Sweden, Slovenia, Switzerland, Finland, and Netherlands. Middle Eastern countries contributed minimally including; Syria, Iran, Israel, UAE, Morocco, Egypt, and Saudi Arabia. Power scoring of e-markets revealed that the top five markets were; AlphaBay, Agora, Nucleus, Abraxas, and Hansa. The most common categories of NPS on these markets were; cannabis and cannabimimetic $\left(1^{\text {st }}\right)$, stimulants $\left(2^{\text {nd }}\right)$, empathogens $\left(3^{\text {rd }}\right)$, and psychedelics $\left(4^{\text {th }}\right)$.

Conclusion: The e-commerce activities on the deep web and the darknet e-marketplace represent an integral component of the NPS e-phenomenon. Unfortunately, recent attempts to examine and study those unlawful activities are outdated. Hence, to achieve real-time and reliable data, the inclusion of data mining tools and knowledge discovery in databases are critical to ensuring a future victory.

Keywords: Deep Web, Darknet, AlphaBay, Valhalla, HANSA, Acropolis, Tochka, Market Power, Tor Browser, Bitcoin payment, social science

\section{Background}

The phenomenon of New psychoactive substances (NPS) growth is escalating exponentially on both divisions of the web; surface and deep (Al-Imam et al., 2016; Al-Imam et al., 2017). This logarithmic spread has also been paralleled by numerous attempts from NPS researchers to counteract its public, health, and economic threats. These attempts have increased from 2010 onward, but they are still partially incapable of facing the "ballooning" progression of the phenomenon; one of the main reasons for that is the anonymous e-trade (e-commerce) activity on the infinite virtual space of the deep web (Chaabane et al., 2010; Mobasher et al. 2001). Several e-markets exist on the deep web, primarily on the darknet which is accessible by means of dedicated search engines from any spot in the world (Biddle et al., 2002; Buxton \& Bingham, 2015; Flick et al., 2013). These e-markets include; the renowned AlphaBay, Agora, Nucleus, Valhalla, and dozens more (Laura \& Me, 2015; Van Buskirk et al., 2016).

Several techniques have been used by researchers to observe and analyse the e-trade on the deep web and its darknet; most were relying either on the observational cross-sectional and retrospective analysis; the most 
common analytic tool for examining the deep web is known as the Internet snapshot method. However, this technique is not only time-consuming, having modest accuracy, and of low level-of-evidence, but it also requires the dedication of time and financial resources (Pastor-Satorras et al., 2001; Siddiqi et al., 2015). Therefore, in this study, a strive to perfect the internet snapshot will be enacted to; enhance the accuracy of the snapshot method (1), improve the generated level-of-evidence (2), reduce time and efforts (3), implement the application of inferential statistics (4), and discuss the potential use of data mining technologies and knowledge discovery in databases (5) (Berry \& Linoff, 1997; Fayyad et al., 1996).

This study is a cross-breed made of an integrative observational analysis of Google Trends database and the e-markets on the darknet. The aims are to; extrapolate the interest of surface web users in the deep web and its incognito e-markets, infer the geographic mapping (countries) of those surface web users, quantify the e-markets on the darknet by assessing each e-market's power (authority), and to infer the most popular categories of NPS on these e-markets. Pertinent geo-mapping of the Middle East and the Arab world will be accompanying each of these steps. A comparison between the European Union and the Middle East will also be made to estimate the proportional contribution of each in connection with the magnitude of the e-trade activities on the darknet.

\section{Materials and Methods}

The e-trade of NPS on the deep web has been assessed via observing the anonymous e-markets on the deep web; this systematic analysis has been initiated by studying the surface web users' interest (attentiveness) towards the deep web and its darknet e-marketplace. This has been assessed by observing the time-related (chronological) changes of five keywords on Google Trends; the keywords are Deep web, Darknet, Tor, Bitcoin network, and Bitcoin (Google, 2017). These keyword terms are representative for the deep web, the darknet, the Tor web browsers, and the Bitcoin payment system (McCoy et al., 2008; Reid and Harrigan, 2013). The historical analysis (2012-2016) of keywords was followed by geographic mapping (geo-mapping) in an attempt to; localise surface web users of the high tendencies to use the deep web and its e-markets (1), and assess the contribution of users from the Middle East and Arabic country, if any exist (2). This analysis is observational and retrospective (longitudinal).

Analysis of the majority of contributing e-markets on darknet was done; these e-markets include Hansa, Darknet Hero League, AlphaBay, Agora, Nucleus Market, Majestic Garden, Real Deal Market, Oasis, Abraxas, Outlaw Market, Middle Earth, Silkkitie, Oxygen, Tochka Market, and Arsenal (Biddle et al., 2002; Al-Imam, 2017; Al-Imam et al., 2017). Assessing each market relied on the concept of the individual basis of power (authority) implemented in social sciences (French et al., 1959; Spekman, 1979). Each e-market has been assessed for its power by analysing three indices; overall rating (1), support rating (2), and the number of votes (3); these data were retrieved from the Grams search engine of the darknet (Laura and Me, 2015; Nakov, 2005). Tabulation of the three power indices was followed by a digital correction (standardisation) and mathematical calculation of a power score unique for each e-market. Hence, the analysis is observational, and it is based on an Internet snapshot which was taken on the $9^{\text {th }}$ of February 2017.

The $3^{\text {rd }}$ and final step was the analysis of the advertised NPS on five of the most popular e-markets; the e-markets are; AlphaBay, Valhalla, HANSA, Acropolis, and Tochka; these e-markets were randomly selected using a random number generator. The e-trade of NPS on the darknet was usually classified into eight categories or less (depending on the e-market). The categories included; Benzodiazepine (1), Cannabis, Hashish, and Cannabimimetics (2), Dissociative substances (3), Empathogens including ecstasy (4), Opioids (5), Prescription-related substances (6) Stimulants and psychostimulants (7), and Psychedelics (8). A subsequent analysis involved the substances enlisted under each of these categories. This step ( $3^{\text {rd }}$ step) led to an extrapolation in relation to the most popular (trending) categories of NPS on darknet e-markets, which was preceded by identifying the most influential (dominant) e-markets on the darknet.

Each of the three analytic steps was paralleled by statistical analyses (descriptive and inferential); statistical inference implemented several tests including; the analysis of variance and covariance (ANOVA), Student's t-test (paired and unpaired), regression models, and nonparametric test. Alpha $(\alpha)$ value of 0.05 and a confidence interval of $95 \%(95 \% \mathrm{CI})$ was set as the cutoff margin for statistical significance. Descriptive statistics were endeavoured to; point out unique data events (statistical outliers), and to explain why these events occurred. Geo-mapping will also be implemented for the region of the Middle East, Arabic Countries, and the European Union. 


\section{Results and Discussion}

Analyses of Google Trends database appears to be steady to some degree (Figure 1) with an exception for the keyword Bitcoin; attentiveness to this keyword seems to soar during 2013-2014 and specifically in March-April 2013, October 2013-March 2014, and December 2016-January 2017. The highest peak for Bitcoin was reached in November 2013. Descriptive statistics clearly shows that the surface web users are highly interested in two main keywords; Tor (17.7 +/- 2.1) and Bitcoin (17.5 +/- 14.9), while the other keywords averaged; Deep web $(5.9+/$ 2.7), Darknet (0.9+/- 1), and Bitcoin network (1.3 +/- 1.5).

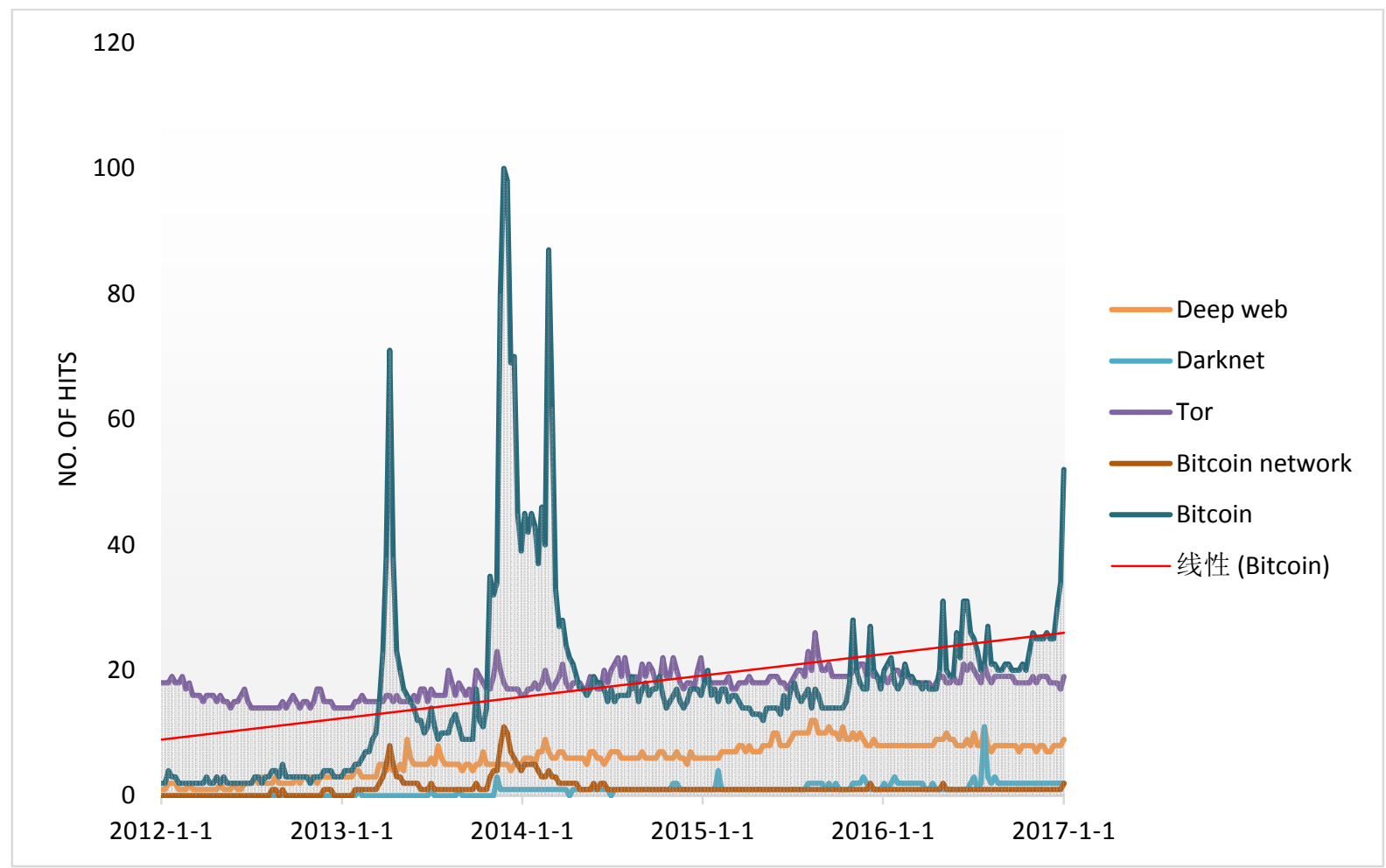

Figure 1. Google Trends: Five Keywords (2012-2016)

The attentiveness towards the keyword Bitcoin network was noticed to be synchronised with the attentiveness towards the keyword Bitcoin; both keywords peaked at the end of November 2013. It is important to know that In October 2013, the Chinese Baidu search engine allowed its users to implement the Bitcoin payment system. Furthermore, in November, it was estimated that approximately 12 million bitcoins were mined in e-payments, and remarkably by the $23^{\text {rd }}$ of November 2013, the total market of bitcoins in the United States has exceeded 10 billion US dollars (Chang G, 2013; Fensch J, 2014; Hendrickson et al., 2015). On the other hand, attentiveness towards the keyword Deep web increased from May till November 2015; it reached a peak in August 2015. The other two keywords, Darknet and Tor, appears to more steady; the keyword Darknet was found to be of the least importance for surface web users. The soaring attentiveness of surface web users towards particular keywords (Tor, Bitcoin, and Deep web) was also validated via Boxplot presentation (Figure 2); statistical outliers were also evident in relation to the keyword Bitcoin. 


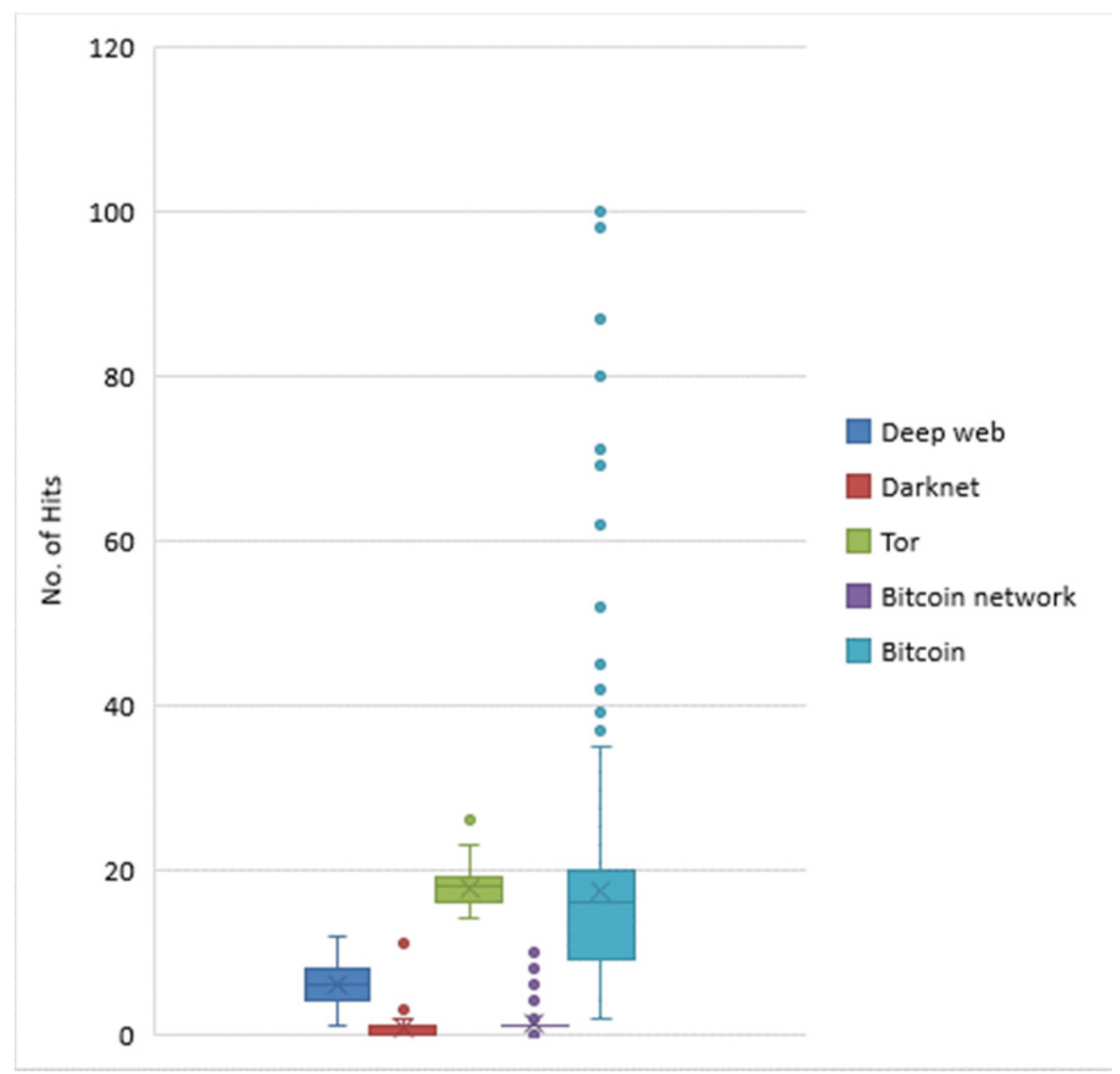

Figure 2. Keywords Mapped on Google Trends: Boxplot Presentation

ANOVA test confirmed the existence of a significant difference ( $p$-value $<0.001)$ in relation to the attentiveness of surface web users towards the five keywords for the period 2012-2016. Further, the independent t-test (Figure 3) revealed that both Tor and Bitcoin Keywords were of comparable popularity (17.7 versus 17.5, $\mathrm{p}=0.419)$ for the period 2012-2016. However, the attentiveness of surface web users towards the keyword Deep web was significantly less than that of; Tor (5.9 versus 17.7, $\mathrm{p}<0.001$ ) and Bitcoin (5.9 versus $17.5, \mathrm{p}<0.001)$. On the other hand the chronological analysis of the mapped keywords has confirmed the presence of significant changes in between 2013 and $2015(\mathrm{p}<0.001)$, while no major shifts in the trends for the keywords between; 2013 and 2014 (0.158), 2013 and 2015 (0.422), and 2014 and 2016 (0.274). 


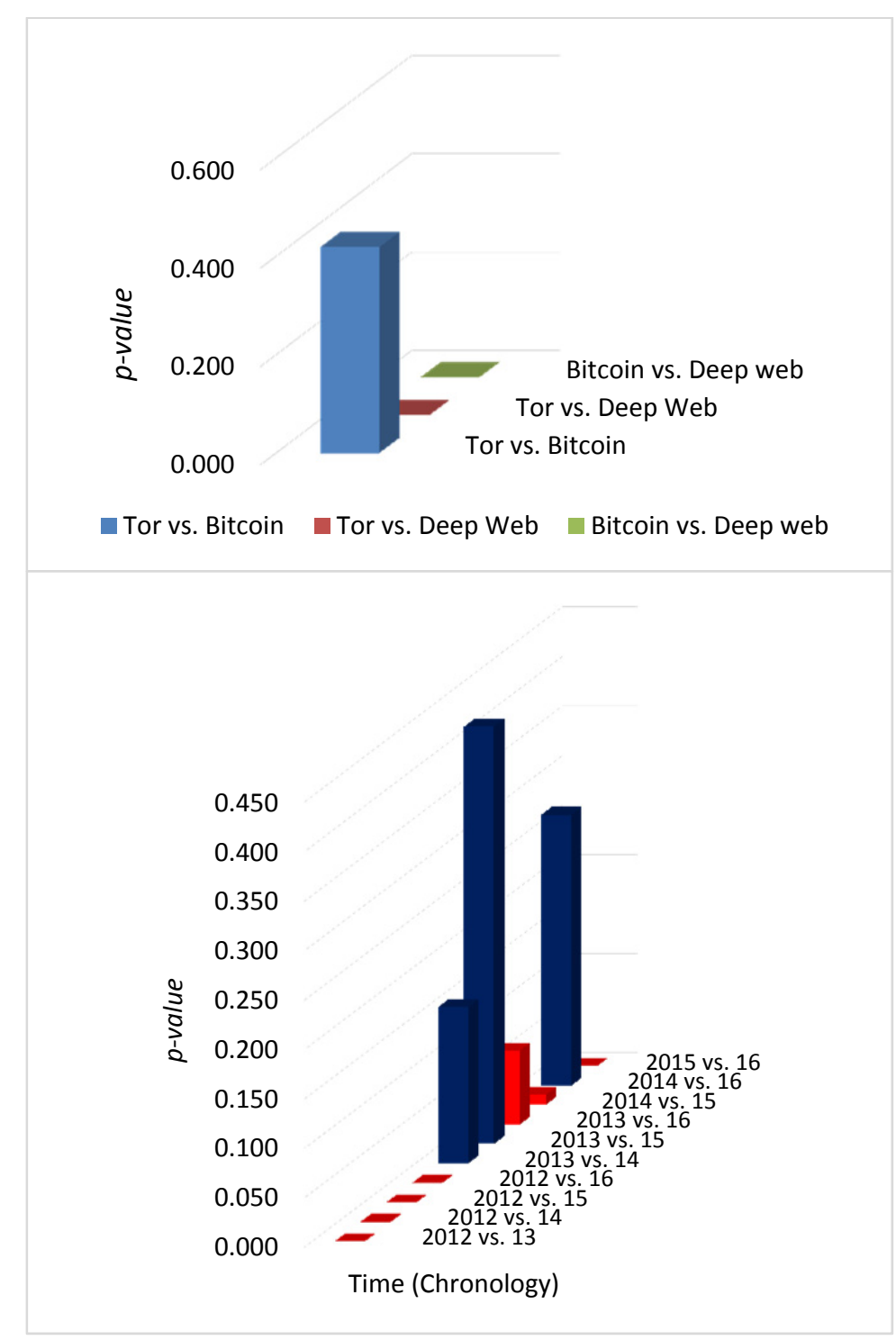

Figure 3. Inferential Statistics: Keywords Mapping on Google Trends

Geo-mapping of the keywords showed that the contribution of five countries was phenomenal (statistical outliers); these countries are (descending order) were Bangladesh, Norway, Germany, Denmark, and Ghana (Figure 4). Other top contributing countries included; Austria, Nigeria, Sweden, Pakistan, Switzerland, Finland, Netherlands, Bolivia, Poland, the Czech Republic, US, UK, and Canada. Apparently, Bangladesh and some African countries are in the top of the list which may be attributed to the ban of social media networks in those countries leading to the use of the anonymous deep web as an alternative "hacking" solution to access the social media, including Facebook and Twitter (Aliens, 2017). Middle Eastern and Arabic countries included (descending order); Syria, Iran, UAE, Israel, Algeria, Morocco, Saudi Arabia, and Egypt (Figure 4). This contribution represented a tiny fragment $(7.1 \%)$ of the global geo-mapping. Concerning the geo-mapped countries, linear regression has confirmed a positive linear correlation between two the keywords Tor and Bitcoin $\left(R^{2}\right.$ score $\left.=0.044\right)$, which has further confirmed that the trends of these two keywords were synchronised for the period 2012-2016. On the other hand, there was some degree of negative correlation between Tor keyword and; Bitcoin network (0.016) and Darknet (0.044). 

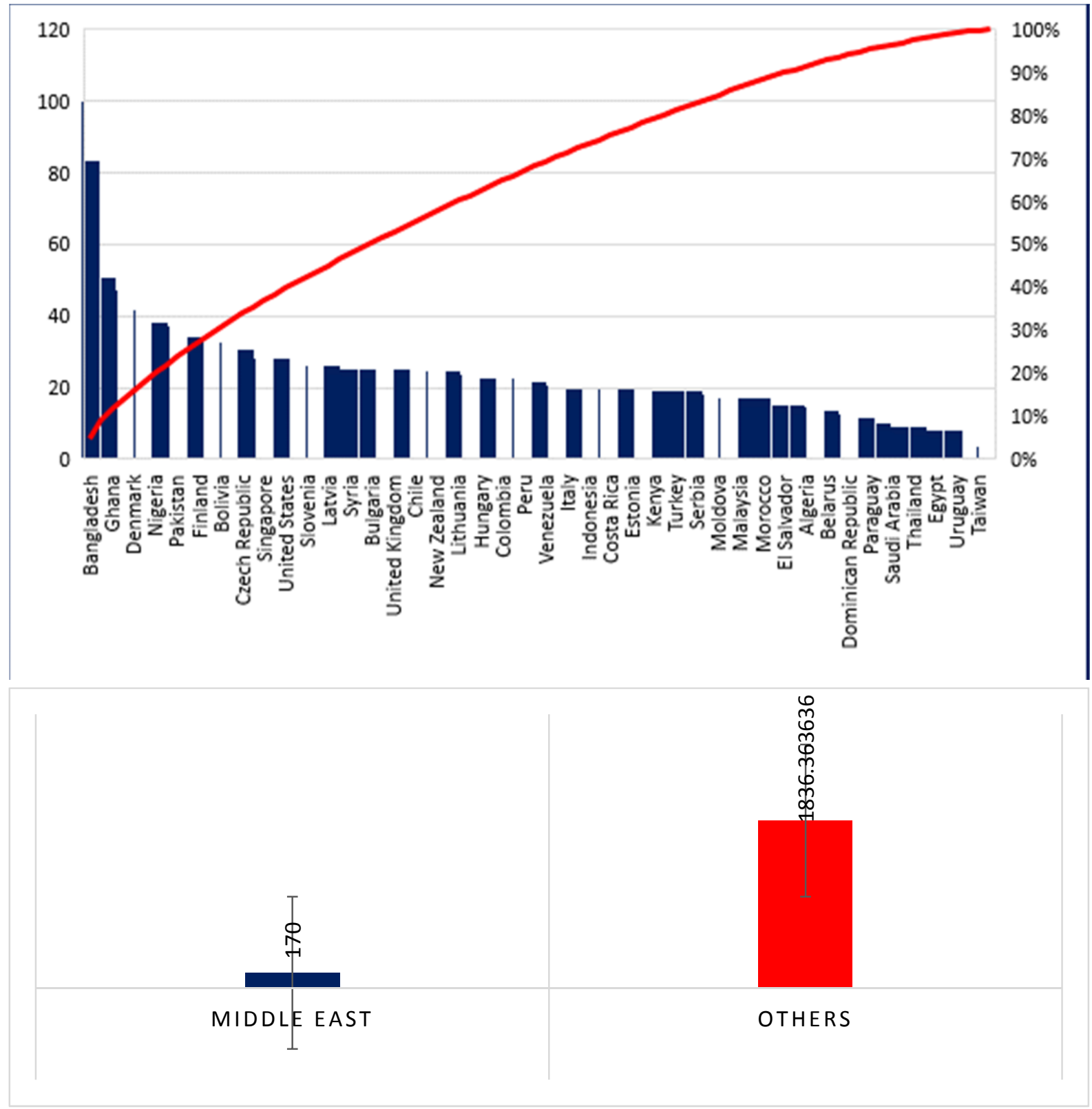

Figure 4. Geo-mapping of the Keywords on Google Trends (2012-2016): Contributing Countries in Descending order (above), Juxtaposition of Middle Eastern Contribution (below)

A comparison was also set between countries from the European Union to infer the existence of significant differences, Norway was an apparent statistical outlier. Student's t-test (independent) was used for statistical inference (Figure 5). Accordingly, it was found that the Middle East contributed much less $(p$-value $<0.001)$ than countries from the EU. Hence, the attentiveness of EU surface web users was the highest in relation to the mapped keywords for the period 2012-2016. Norway (rank $1^{\text {st }}$ ) and Germany (rank 2nd) represented the top contributing countries from the EU (Figure 6). 


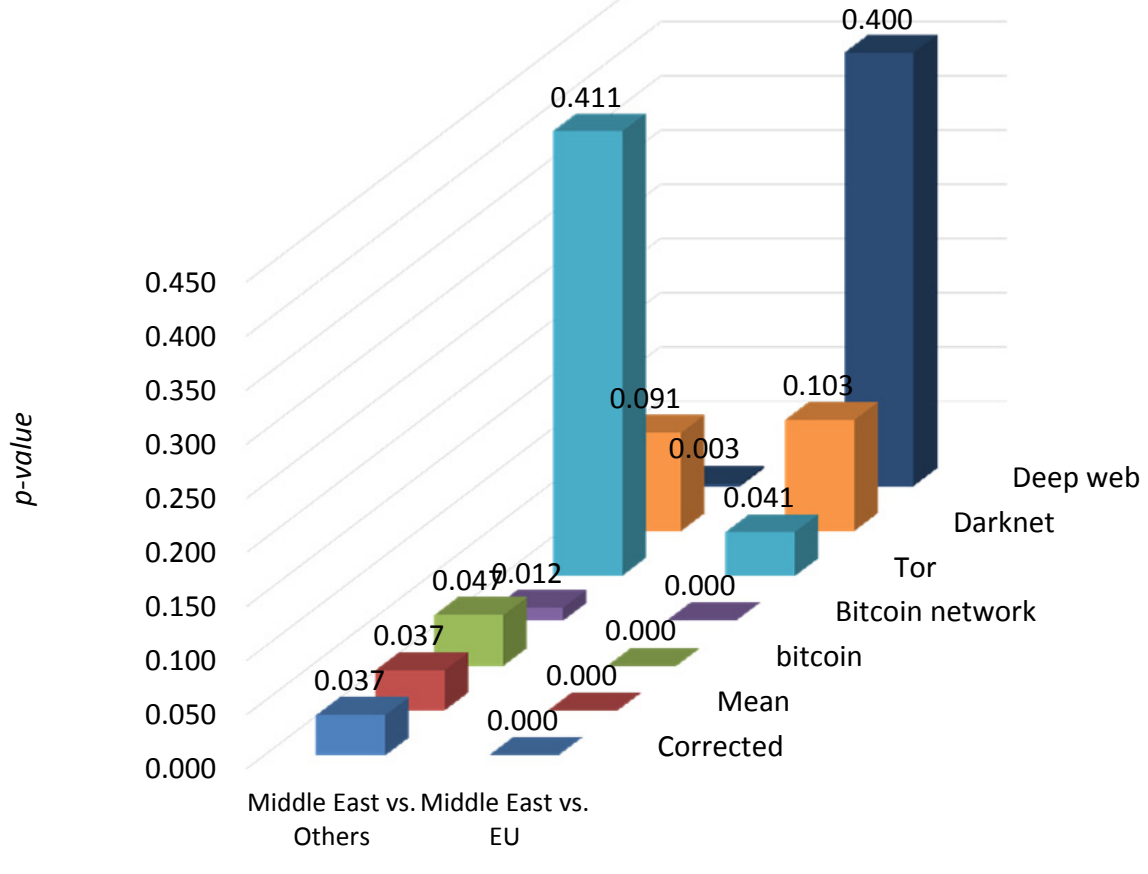

Figure 5. Inferential Statistics of Regional Geo-mapping: The Middle East and the European Union

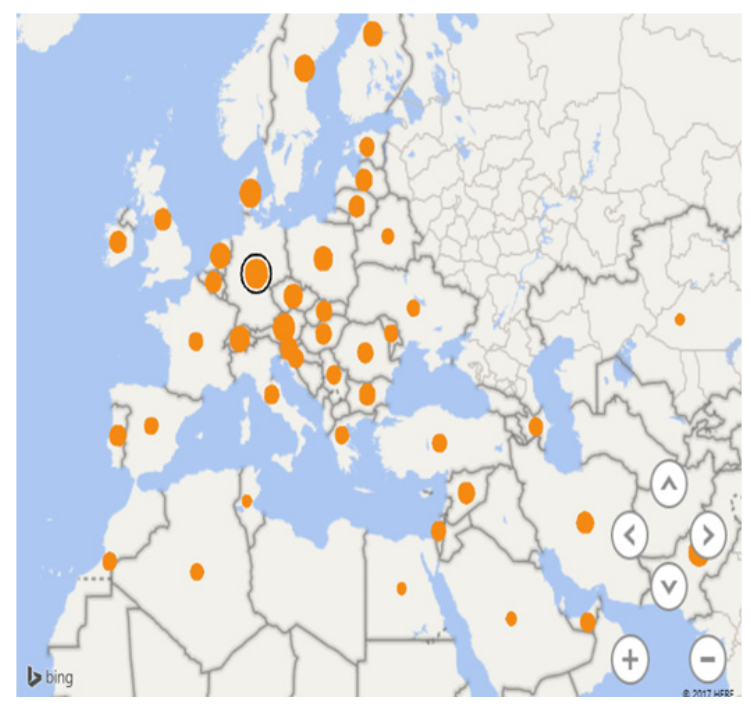

Figure 6. Geo-mapping of Keywords on Google Trends: Middle East, Arabia, and the European Union

On the darknet, fifteen ( $\mathrm{n}=15)$ major e-markets have been identified; AlphaBay, Agora, Nucleus Market, Abraxas, HANSA, Middle Earth, Darknet Hero League, Outlaw Market, Majestic Garden, Silkkitie, Oasis, Real Deal Market, Tochka Market, Oxygen, and Arsenal (Google, 2017). The power score analysis revealed two outliers (exceptionally attractive) e-markets; AlphaBay and Agora (Figure 7). It seems that AlphaBay is the principal market on the darknet. Descriptive Statistics (Table 1, Figure 8) naturally visualise that the top three e-markets (AlphaBay, Agora, and Nucleus Market) are in the lead with a big gap over other e-markets. 
Table 1. Power Scoring of e-markets on the Darknet

\begin{tabular}{llcccc}
\hline & Darknet Market & Overall Rating & Support Rating & Votes & Power Score (Corrected) \\
\hline 1 & AlphaBay & 4 & 4 & 243 & 225.49 \\
2 & Agora & 4 & 4 & 192 & 192.16 \\
3 & Nucleus Market & 4 & 4 & 152 & 166.01 \\
4 & Abraxas & 4 & 3 & 82 & 111.93 \\
5 & Hansa & 4 & 4 & 51 & 100.00 \\
6 & Middle Earth & 4 & 3 & 55 & 94.28 \\
7 & Darknet Hero League & 4 & 4 & 34 & 88.89 \\
8 & Outlaw Market & 4 & 4 & 27 & 84.31 \\
9 & Majestic Garden & 4 & 4 & 14 & 75.82 \\
10 & Silkkitie & 4 & 3 & 26 & 75.33 \\
11 & Oasis & 4 & 4 & 11 & 73.86 \\
12 & Real Deal Market & 4 & 4 & 7 & 71.24 \\
13 & Tochka Market & 3 & 4 & 8 & 63.56 \\
14 & Oxygen & 3 & 3 & 8 & 55.23 \\
15 & Arsenal & 2 & 1 & 10 & 31.54 \\
\hline
\end{tabular}

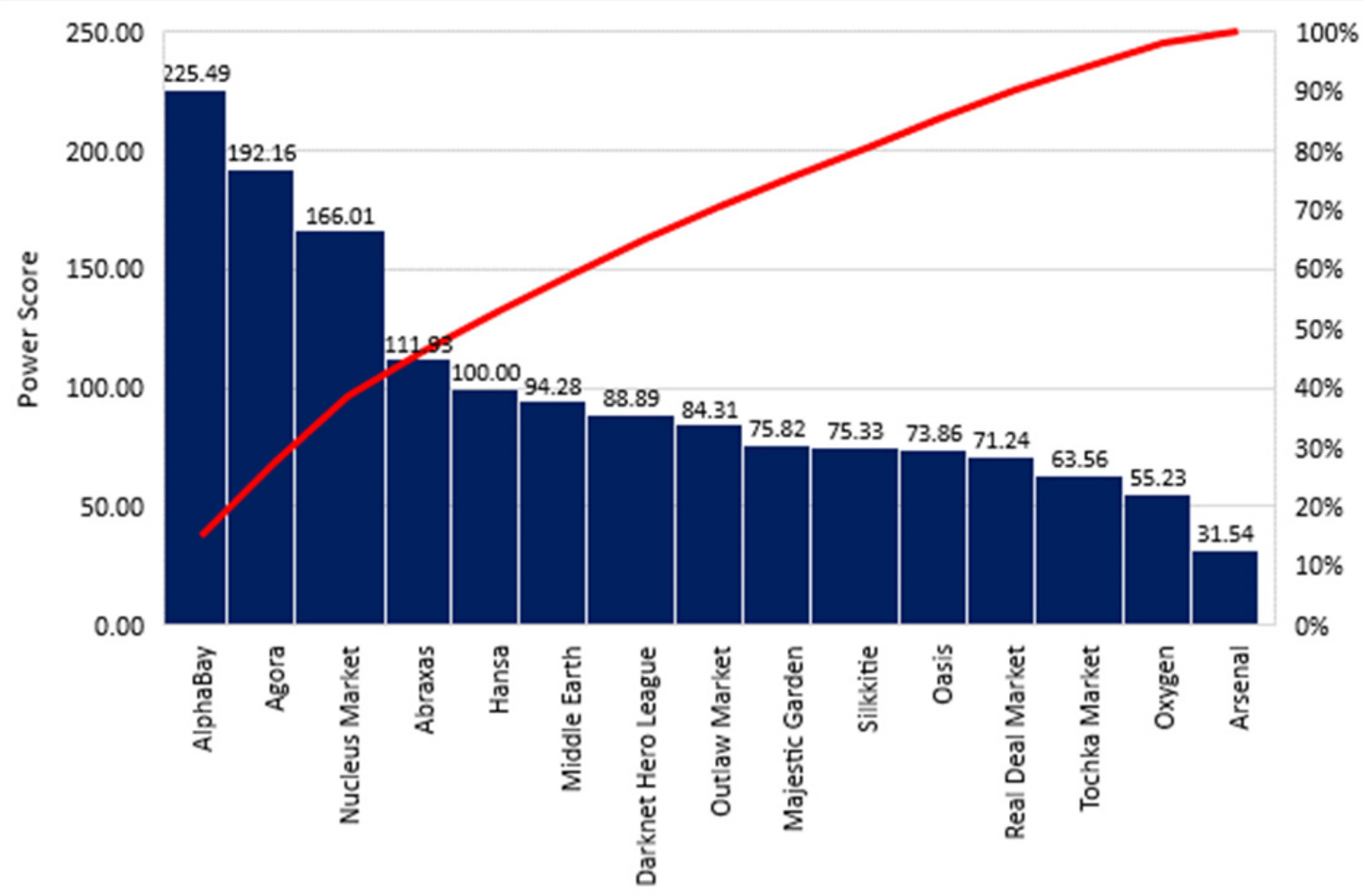

Figure 7. Power Score of e-markets on the Darknet: Pareto Chart. 
300

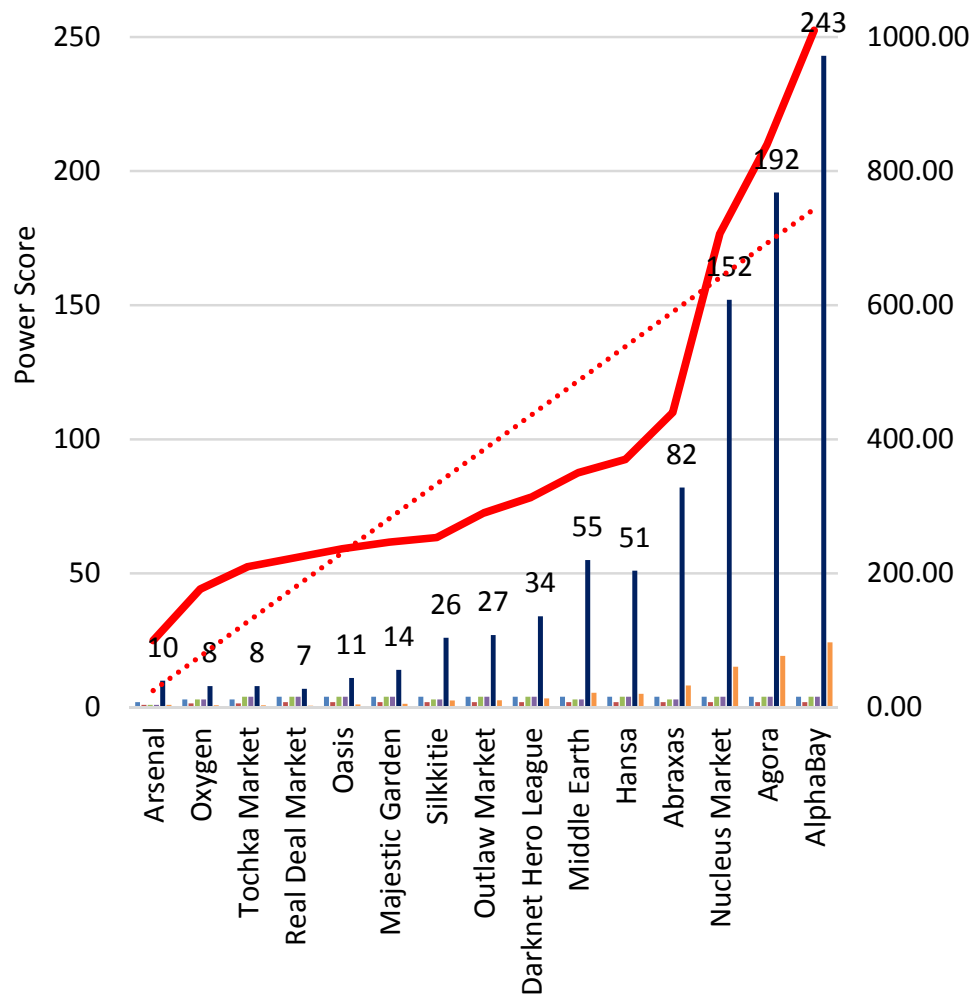

1200.00

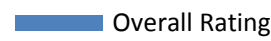

Corrected

Support Rating

Corrected2

Votes

Corrected3

Power Score (Corrected)

线性 (Power Score (Corrected))

Figure 8. Power Scoring of e-markets on the Darknet

Inferential analysis, using linear regression, shows a positive linear correlation between; power score and the number of votes $\left(R^{2}\right.$ score $\left.=0.961\right)$, overall rating and support rating for each e-market $\left(R^{2}=0.549\right)$. Furthermore, the power score was rearranged in descending order to permit the analysis of high-power versus low-power e-markets. Initially, a comparison was made between the top seven markets and the remaining eight e-markets (Figure 9), which showed a clear leverage of the top seven e-markets ( -value $=0.006)$. Even when comparing the strongest two e-markets versus the remaining 13 e-markets, AlphaBay and Agora were still proven superior $(\mathrm{p}=0.018)$. 


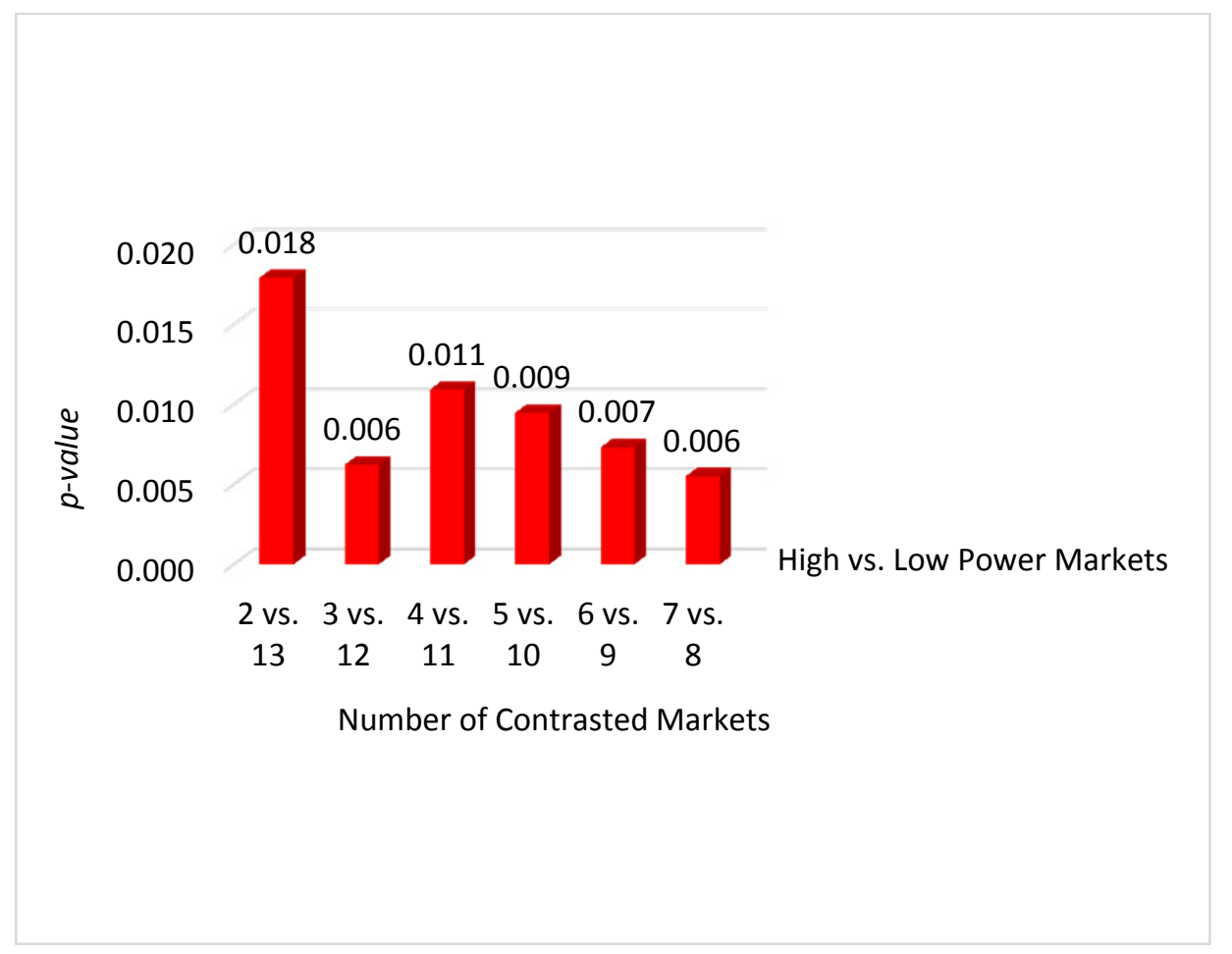

Figure 9. Inferential Statistics: Analyses of High-power versus Low-power e-markets

The final analytic step was to pick five e-markets from the darknet and perform an observational analysis of the advertised NPS (Figure 10 and 11). A random number generator was used, the five e-markets were; AlphaBay, Valhalla, HANSA, Acropolis and Tochka. The randomization in selection aims to; prevent selection bias, and to assess the heterogeneity of advertised substances (NPS) within e-markets of different power-scoring. The Internet snapshot was taken on the $25^{\text {th }}$ of February 2017. Categories of NPS found on AlphaBay were; Benzodiazepine (14860 items), Cannabis and Hashish (65275), Dissociatives (4319), Ecstasy (30207), Opioids (16889), Prescription (8736), Stimulants (33098), and Psychedelics (14007). Valhalla categorised the NPS as; Cannabis and Hashish (3917), Stimulants (2266), Empathogens (2108), Psychedelics (1065), Opioids (944), Prescription (1766), Dissociatives (258), and Depressants (53). HANSA e-market advertised NPS as; Cannabis and Hashish (4796), Opioids (535), Psychedelics (1313), Prescription (1345), Stimulants (2582), Ecstasy (2780), Benzodiazepine (730), and Dissociatives (360). On the other hand Acropolis e-market adopted a bit different categorization system; Benzodiazepine (3), Cannabis and Hashish (186), Dissociatives (6), Ecstasy (52), Opioids (34), Prescription-related (12), Psychedelics (59), Stimulants (52), Research Chemicals (3), and Barbiturates (1). The $5^{\text {th }}$ e-market, Tochka, categorised NPS in a classical way; Cannabis (108), Stimulants (53), Psychedelics (21), Empathogens (17), Opioids (7), Dissociatives (6), Prescription (44), and Others (20). 


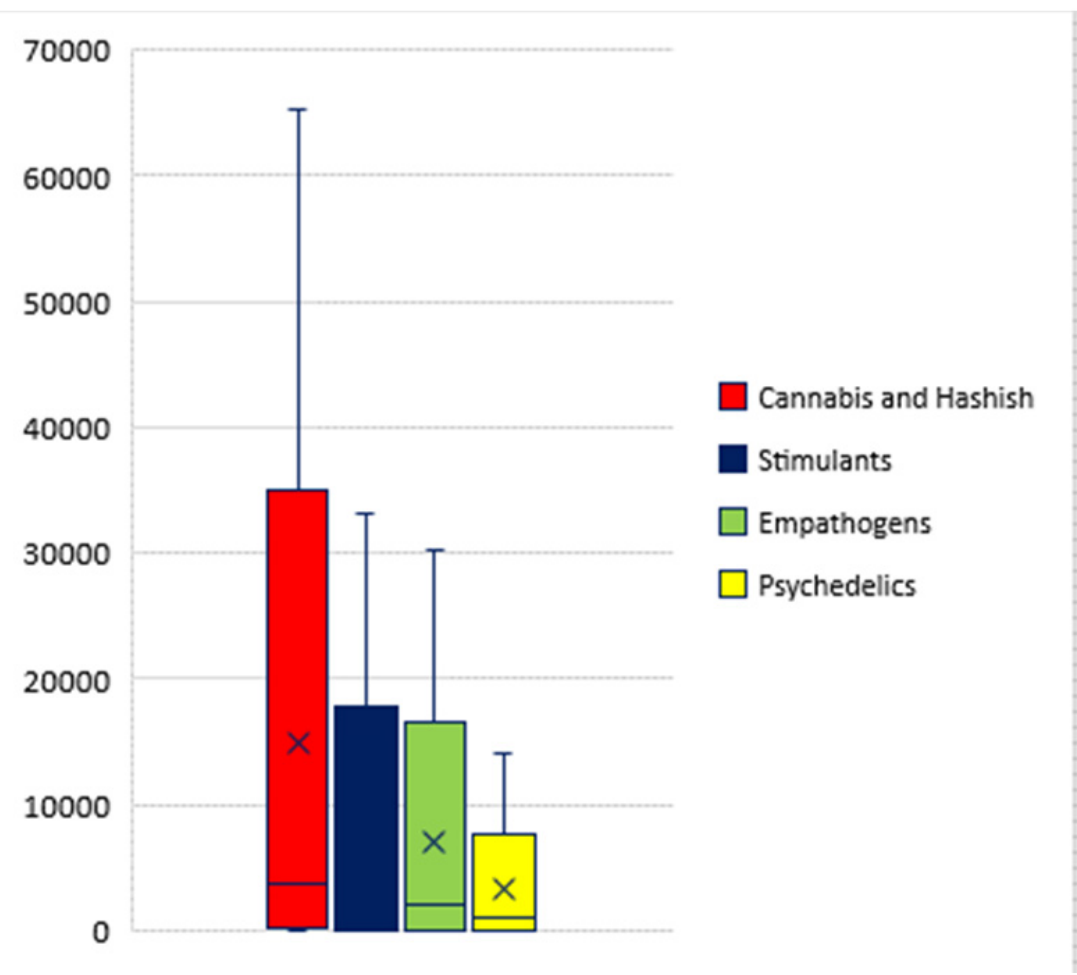

Figure 10. Most Popular Categories of NPS on the Darknet

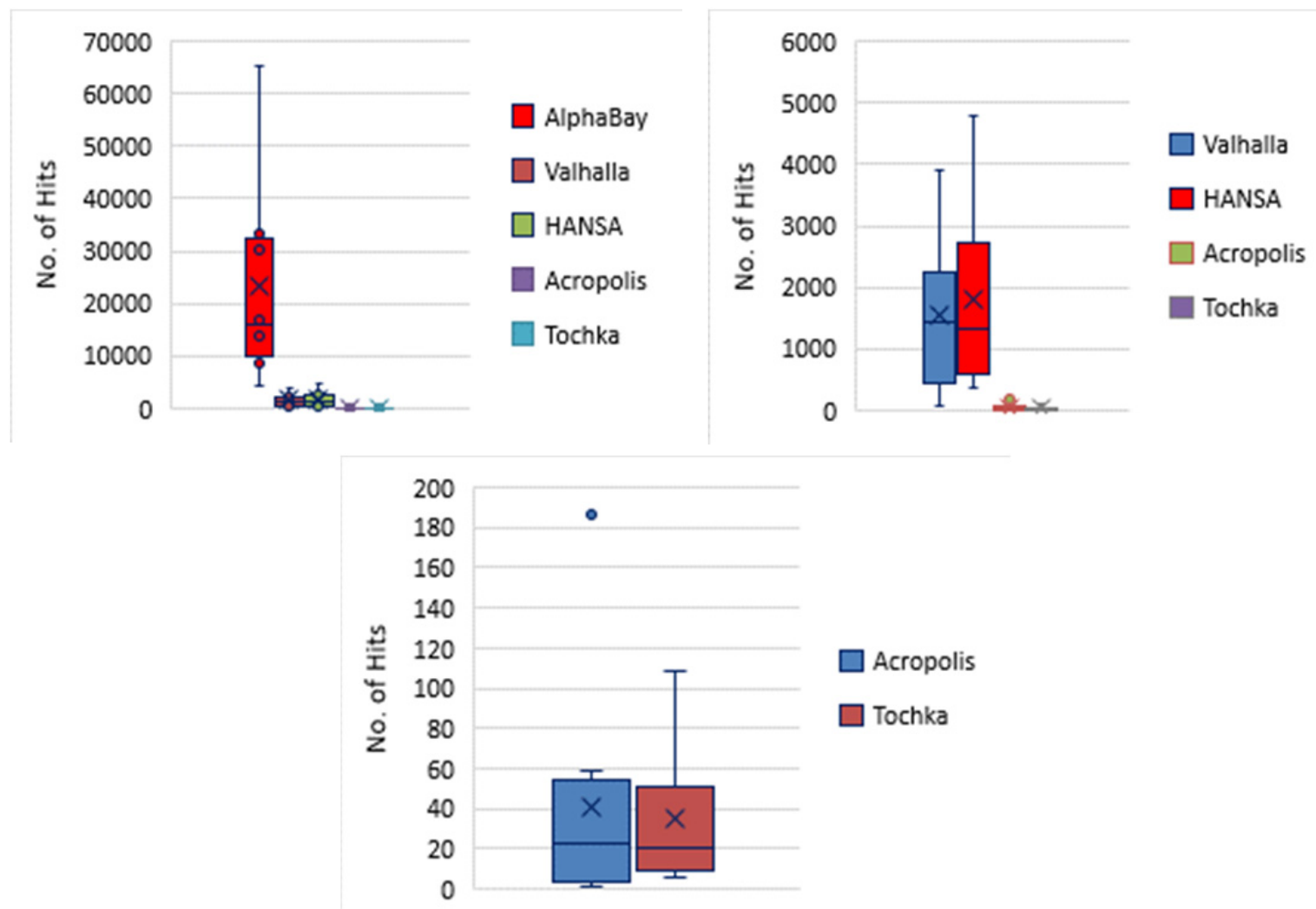

Figure 11. The Total Number of Substances Advertised on the Selected e-markets.

It seems that four categories of NPS dominated each of these five e-markets, these classes are; cannabis-hashish and cannabinoids (rank $1^{\text {st }}$ ), Stimulants (rank $2^{\text {nd }}$ ), empathogens ( $\left.\operatorname{rank} 3^{\text {rd }}\right)$, and psychedelics $\left(\operatorname{rank~} 4^{\text {th }}\right)$. 
Furthermore, the number of items, under the category of cannabis and cannabinoids, behaved as a statistical outlier in Acropolis e-market; cannabis and cannabinoids have contributed to $45.6 \%$ of the total number of substances advertised within the Acropolis e-market. However, inferential statistics revealed that there was no statistically significant difference in the number of items which were sold under each of the top four NPS categories (Figure 12). On the other hand, there was a significant difference in relation to the number of items advertised on all of the randomly selected e-markets (Figure 13), with an exception for the number of substances on Acropolis and Tochka e-markets ( $p$-value $=0.09)$. It is to be deducted that AlphaBay e-market is always in the lead, while cannabis and cannabimimetic represent the most popular category of NPS in circulation within the darknet e-marketplace.

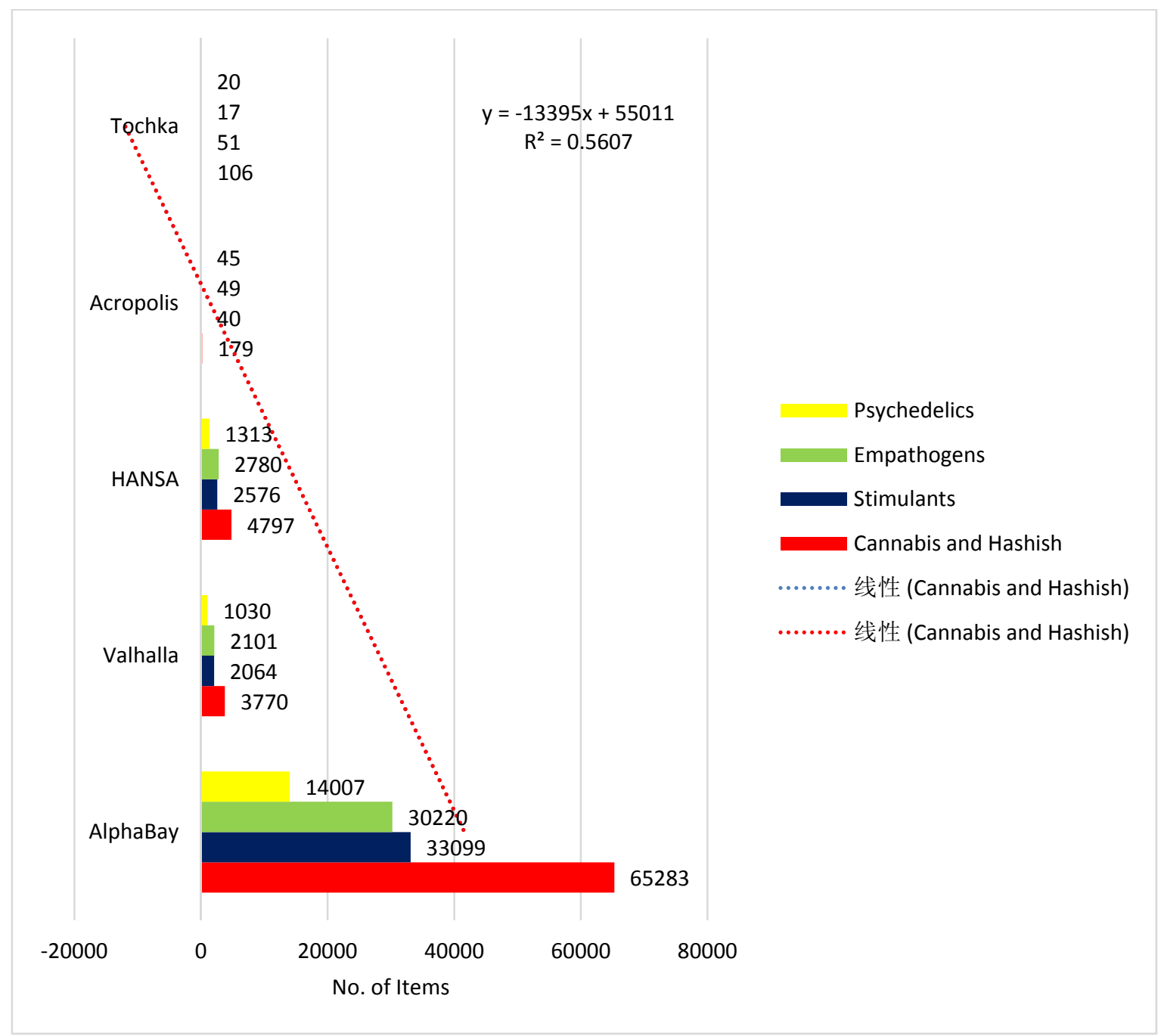

Figure 12. Most Popular Categories of NPS on the Darknet e-markets 


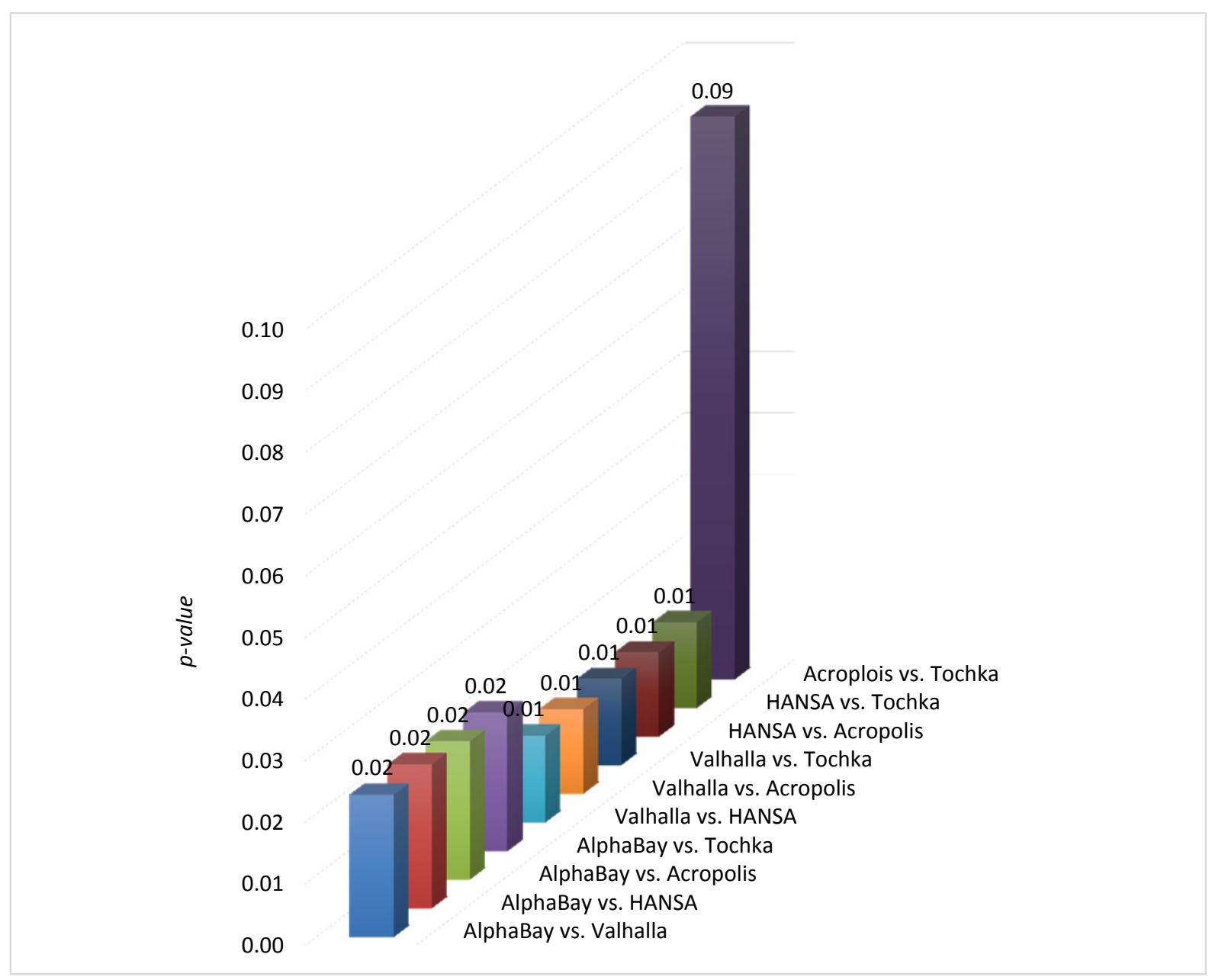

Figure 13. Inferential statistics: Most Dominant e-markets

A systematic analysis was finally done for the substances advertised under each category of NPS in AlphaBay e-market. These substances included; LSD, shrooms, DMT, Mescaline, LSA, DMA / DOX, NBOMe, 2C-B, and other research chemicals/RCs (Psychedelics); Speed, Meth, Adderall and Vyvanse, 2-FA, Pressed Pills, Cocaine, Crack, RCs, and others (Stimulants); Prescription, Fentanyl and RCs, Heroin, Pills, Prescription-related, and others (Opioids); MDMA, MDA, Methylone, and others (Empathogens); Ketamine, MXE, GHB, and others (Dissociatives); RCs and combinations, Pills, Powder, and others (Benzodiazepines). Cannabis and Hashish included diverse items classified as; buds and flowers (38122), shakes (635), Concentrates (8081), Hash (8455), Prerolls (243), Edibles (2059), Topical and others (228), Seeds (1098), Synthetic Cannabinoids and Cannabimimetics (551), and others (5811). In summary, the top advertised substances on AlphaBay were; Cannabis and Hashish (65283), Cocaine (13291), MDMA (11599), methamphetamine (5791), and LSD (6489). These substances accounted for $54.7 \%$ of the total output of AlphaBay e-trade events. 


\section{Conclusion}

The deep web is not only anonymous but also an enormous virtual place in which an observer can quickly be overwhelmed while conducting an observational analysis or taking a snapshot. However, this study implemented a tactical approach to render the task; easier, more focused, and made of as minimum observations as possible. The tactic is made of three overlapping steps, the $1^{\text {st }}$ one relies on Google trends analyses to estimate the interest (attentiveness) of surface web users in the deep web, and the geo-mapping of the contributing countries including those from the Middle East and the Arab World. The $2^{\text {nd }}$ and $3^{\text {rd }}$ steps are carried out on the deep web itself to infer; the basis of power for the e-markets on the darknet, and the most common NPS on the e-marketplace. AlphaBay and Agora were found to be dominating the e-marketplace, while the most frequently sold categories of NPS included cannabimimetic, stimulants, empathogens, and psychedelics. This simplified yet systematic Internet snapshot technique can be repeated at any given moment of time. Besides, when this method is combined with powerful tools for data mining, the data output should be highly accurate, and in real-time; the reduction in time and efforts should be remarkable.

\section{Competing Interests Statement}

The authors have nothing to be declared.

\section{Source of Funding}

This study has been entirely self-funded by the authors.

\section{References}

Aliens C. Nigeria Lacks a 2017 Cybersecurity Strategy but Faces the Most Cybercrime Ever Seen. Retrieved 10 March, 2017 ,

from https://www.deepdotweb.com/2017/01/06/nigeria-lacks-2017-cybersecurity-strategy-faces-cybercrime-everseen/

Al-Imam, A. (2017). Could Hallucinogens Induce Permanent Pupillary Changes in (Ab) users? A Case Report from New Zealand. Case Reports in Neurological Medicine, 2017. https://doi.org/10.1155/2017/2503762

AL-Imam, A., Santacroce, R., Roman - Urrestarazu, A., Chilcott, R., Bersani, G., Martinotti, G., \& Corazza, O. (2017). Captagon: use and trade in the Middle East. Human Psychopharmacology: Clinical and Experimental, 32(3).

Al-Imam, A., Simonato, A. P., \& Corazza, O. (2016). Haloperidol, an old antipsychotic with potential use by NPS users in Iraq. Research and Advances in Psychiatry, 3(3), 81-84. Retrieved from https://www.rapjournal.eu/materiale_cic/948_3_3/8031_haloperidol/article.htm

Berry, M. J., \& Linoff, G. (1997). Data mining techniques: for marketing, sales, and customer support. John Wiley $\&$ Sons, Inc..

Biddle, P., England, P., Peinado, M., \& Willman, B. (2002, November). The darknet and the future of content protection. In ACM Workshop on Digital Rights Management (pp. 155-176). Springer, Berlin, Heidelberg.

Buxton, J., \& Bingham, T. (2015). The rise and challenge of dark net drug markets. Policy Brief, 7.

Chaabane, A., Manils, P., \& Kaafar, M. A. (2010, September). Digging into anonymous traffic: A deep analysis of the tor anonymizing network. In Network and System Security (NSS), 2010 4th International Conference on(pp. 167-174). IEEE. https://doi.org/10.1109/NSS.2010.47

Chang, G. G. (2013). A China Triangle: Bitcoin, Baidu and Beijing.

Fayyad, U., Piatetsky-Shapiro, G., \& Smyth, P. (1996). From data mining to knowledge discovery in databases. $A I$ magazine, 17(3), 37.

Flick, C., \& Sandvik, R. A. (2013). Tor and the darknet: researching the world of hidden services. In Proceedings of the thirteenth international conference, the possibilities of ethical ict (pp. 150-157).

French, J. R., Raven, B., \& Cartwright, D. (1959). The bases of social power. Classics of organization theory, 7.

Google. Google Trends. Retrieved 10 March, 2017, from https://www.google.com/trends/explore?date=2012-01-01\%202017-01-01\&q=Deep\%20web,Darknet,\%2F m\%2F04n38z,\%2Fm\%2F0vp7t27,\%2Fm\%2F05p0rrx

Hendrickson, J. R., Hogan, T. L., \& Luther, W. J. (2016). The political economy of bitcoin. Economic Inquiry, 54(2), 925-939. https://doi.org/10.1111/ecin.12291 
Laura, L., \& Me, G. (2017). Searching the Web for illegal content: the anatomy of a semantic search engine. Soft computing, 21(5), 1245-1252. https://doi.org/10.1007/s00500-015-1857-4

McCoy, D., Bauer, K., Grunwald, D., Kohno, T., \& Sicker, D. (2008, July). Shining light in dark places: Understanding the Tor network. In International Symposium on Privacy Enhancing Technologies Symposium (pp. 63-76). Springer, Berlin, Heidelberg. https://doi.org/10.1007/978-3-540-70630-4_5

Mobasher, B., Dai, H., Luo, T., \& Nakagawa, M. (2001, August). Improving the effectiveness of collaborative filtering on anonymous web usage data. In Proceedings of the IJCAI 2001 Workshop on Intelligent Techniques for Web Personalization (ITWP01) (pp. 53-61).

Nakov, P., \& Hearst, M. (2005, June). Search engine statistics beyond the n-gram: Application to noun compound bracketing. In Proceedings of the Ninth Conference on Computational Natural Language Learning (pp. 17-24). Association for Computational Linguistics. https://doi.org/10.3115/1706543.1706547

Pastor-Satorras, R., Vázquez, A., \& Vespignani, A. (2001). Dynamical and correlation properties of the Internet. Physical review letters, 87(25), 258701. https://doi.org/10.1103/PhysRevLett.87.258701

Reid, F., \& Harrigan, M. (2013). An analysis of anonymity in the bitcoin system. In Security and privacy in social networks (pp. 197-223). Springer New York. https://doi.org/10.1007/978-1-4614-4139-7_10

Siddiqi, S., Verney, C., Dargan, P., \& Wood, D. M. (2015). Understanding the availability, prevalence of use, desired effects, acute toxicity and dependence potential of the novel opioid MT-45. Clinical Toxicology, 53(1), 54-59. https://doi.org/10.3109/15563650.2014.983239

Spekman, R. E. (1979). Influence and information: An exploratory investigation of the boundary role person's basis of power. Academy of Management Journal, 22(1), 104-117. https://doi.org/10.2307/255482

Van Buskirk, J., Naicker, S., Roxburgh, A., Bruno, R., \& Burns, L. (2016). Who sells what? Country specific differences in substance availability on the Agora cryptomarket. International Journal of Drug Policy, 35, 16-23. https://doi.org/10.1016/j.drugpo.2016.07.004

\section{Copyrights}

Copyright for this article is retained by the author(s), with first publication rights granted to the journal.

This is an open-access article distributed under the terms and conditions of the Creative Commons Attribution license (http://creativecommons.org/licenses/by/4.0/). 